\title{
CDISC SEND Method of Termination Terminology
}

National Cancer Institute

\section{Source}

National Cancer Institute. CDISC SEND Method of Termination Terminology. NCI

Thesaurus. Code C89975.

The terminology that includes concepts relevant to the Clinical Data Interchange Standards Consortium (CDISC) Standard for the Exchange of Non-clinical Data (SEND) method of termination. 